locus of permitted values in the $\gamma-g$ plane. Graphite particles with either ice mantles ${ }^{8}$ or solid hydrogen mantles ${ }^{9}$ could meet this requirement. The latter type of dielectric mantle is, however, preferable for matching the detailed shape of the interstellar extinction curve ${ }^{8,10}$. It is also consistent with the recent observations of Cudaback, Gaustad and Knacke ${ }^{11}$ indicating the lack of a $3 \cdot 1$ micron ice band in highly reddened stars.

It is also worth noting that the properties of diamond with respect to absorption features at $\sim 8$ micron and at $2300 \AA$, referred to by Saslaw and Gaustad ${ }^{1}$, have analogues in the graphite case as well. 'The former wavelength is close to that defined by the Debye frequency of graphite, and the latter wavelength is appropriate to a transition of $\pi$-electrons to the conduction band, as in the case of diamond. There is thus no reason for preferring diamond to graphite in these respects. Graphite particles with dielectric mantles (for example, solid $\mathrm{H}_{2}$ ) seem able to fit most of the modern observations, while diamond particles clearly do not meet these requirements.

N. C. WiCkRAMASINGHW

Institute of Theoretical Astronomy,

University of Cambridge.

Received February 3, 1969.

${ }^{1}$ Saslaw, W. C., and Gaustad, J. E., Nature, 221, 160 (1969).

" Phillipp, H. R., and Taft, E. A., Phys. Rev.,127, 159 (1962).

${ }^{3}$ Wickramasinghe, N. C., Interstellar Grains (Chapman and Hall, Ltd, London, 1967)

4 Johnson, H. L., Astrophys. J, 141, 923 (1965).

s Nandy, K., Pub. Roy. Obs. Edinburgh, 3, 142 (1964).

${ }^{6}$ Stecher, T. P., Astrophys. J.,142, 1683 (1965).

'Witt, A. N., Astrephys. J.,152, 59 (1968).

${ }^{8}$ Van de Hulst, H. C., and de Jong, T., Physica, 41, 151 (1969).

- Wickramasinghe, N. C., and Krishna Swamy, K. S., Mon. Not. Roy. Astro. Soc. (in the press).

${ }^{10}$ Wickramasinghe, N. C., and Nandy, K., Nature, 219, 1347 (1968).

${ }^{11}$ Cudaback, D. D., Gaustad, J. E., and Knacke, R. F., Bull. Amer. Astro. Soc (in the press).

\section{Epicentre Determination by Seismic Arrays}

Lilwall and Douglas ${ }^{1}$ recently described two methods for the determination of epicentres of seismic events, using data from the four UK designed seismic arrays. In their first method they use tables of phase velocity against distance, and for their second method they claim to use "azimuth only". Although I admire the elegance of many-variable non-linear least-squares fits, I wish to draw attention to the fact that, contrary to the claims of the authors, their second method still uses $\mathrm{d} T / \mathrm{d} \Delta$ as a function of distance, even though corrections for the preliminary $\mathrm{d} T / \mathrm{d} \Delta$ are now calculated, along with the epicentre and origin-time corrections. Predictably, the increased number of unknowns in the second method makes the solutions less stable.

Knowing trom experience the frequent frustrations and pitfalls of measuring $\mathrm{d} T / \mathrm{d} \Delta$ from seismic array data and interpreting it as a function of distance, I welcome the suggestion of Lilwall and Douglas for using only the azimuth-determining properties of seismic arrays in epicentre determinations. Then, contrary to usual seismological practice, the use of precise arrival-time data and travel-time tables, together with (preliminary) travel-time derivatives, can be rejected. This avoids error contributions from the measurement of time, from station and source correction terms and from travel-time variations along different paths, and only requires belief in great circle paths for seismic first arrivals. The equations of condition on a spherical Earth become very simply

$$
\delta \alpha_{i}=\frac{\sin \beta_{i}}{\sin \Delta_{j}} \delta x-\frac{\cos \beta_{i}}{\sin \Delta_{i}} \delta y
$$

where $\delta \alpha_{\mathrm{i}}$ are corrections to be applied to event azimuths observed at the arrays, the $\Delta_{i}$ are preliminary epicentral distanees, $\beta_{i}$ are the azimuths of the arrays from the preliminary epicentre, and $\delta x$ and $\delta y$ are north and east corrections to be applied to the preliminary epicentre.

Lilwall and Douglas compare their improved epicentres to our ${ }^{2}$ errors of $300-400 \mathrm{~km}$, obtained by a fully automatic computer search of the data from one array at Yellowknife, based on the apparent direction of energy arrival of seismic wavefronts. Such a method necessarily relies on some type of averaging over the first few signal cycles and therefore is perceptive to small distortions of the signal shape across the array. If this effect is avoided, the arrival azimuths are often surprisingly reproducible and accurate. As an illustration I have chosen the same epicentral region (Kazakh, Central Russia) as Lilwall and Douglas. In Table 1 I list our "best" estimates of arrival azimuths for a number of Kazakh events, together with the best independently available great circle azimuths ${ }^{3}$, and an estimate of the signal-to-noise ratio. The azimuth estimate is the unweighted average of the least-squares azimuths of first peaks and of first troughs; the formal standard deviations of the first-peak solutions are also given. The mean error of $1 \cdot 26^{\circ}$ in the estimated azimuths is significant, but neither geologic nor geometric seismometer site corrections were applied, although they are known to exist. The r.m.s. scatter of the azimuth residuals of less than $1^{\circ}$ amounts to less than $100 \mathrm{~km}$ at the epicentre, however. Using four geographically well located arrays, the r.m.s. error for high signal-to-noise ratio events in this region should be reduced by $\sqrt{ } 3$, bringing it within the limits of accuracy of USCGS-PDE epicentre reports. Distasteful as it may be to seismologists, I therefore recommend to workers with convenient access to multi-array data our simple trigonometrical equation of condition for further minimization studies.

Table 1. AZIMUTH ESTIMATES FOR SEISMIC SIGNALS FROM KAZAKH

$\begin{array}{cc}\begin{array}{c}\text { Great circle } \\ \text { a\%. (deg.) }\end{array} & \begin{array}{c}\text { Approx. signal- } \\ \text { noise ratio* }\end{array} \\ 351 \cdot 1 & 10-12 \\ 351 \cdot 1 & 10-30 \\ 351 \cdot 1 & 20-30 \\ 351 \cdot 1 & 10-20 \\ 351 \cdot 1 & 10-20 \\ 351 \cdot 1 & <10 \\ 351 \cdot 1 & 10 \\ 351 \cdot 1 & 10 \\ 351 \cdot 1 & 10-50 \\ 351 \cdot 2 & 7-10 \\ 351 \cdot 4 & 5-10 \\ 351 \cdot 2 & 10-20 \\ 351 \cdot 2 & 10-20 \\ 351 \cdot 1 & 10-15 \\ 351 \cdot 2 & 10-20 \\ 351 \cdot 2 & 30 \\ 351 \cdot 1 & 40 \\ 351 \cdot 2 & 50 \\ 351 \cdot 2 & 20 \\ 351 \cdot 1 & 2 \text { only }\end{array}$

$$
\begin{gathered}
\begin{array}{c}
\text { Est. az. } \\
\text { (deg) }
\end{array} \\
351 \cdot 4 \\
352 \cdot 2 \\
353 \cdot 0 \\
351 \cdot 9 \\
351 \cdot 8 \\
353 \cdot 3 \\
352 \cdot 7 \\
353 \cdot 4 \\
353 \cdot 4 \\
352 \cdot 2 \\
352 \cdot 1 \\
352 \cdot 6 \\
351 \cdot 8 \\
353 \cdot 0 \\
353 \cdot 4 \\
352 \cdot 1 \\
353 \cdot 0 \\
352 \cdot 8 \\
352 \cdot 0 \\
351 \cdot 1
\end{gathered}
$$

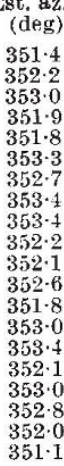

Average actual azimuth residual

Average of formal least squares standard deviations

r.m.s. scatter of azimuth residuals

* The signal-to-noise ratio usually varies between channels; only rough estimates are therefore given.

It is still necessary to associate the ovents measured at a number of arrays with each other unambiguously; the accuracy required to do this is, however, not very high.

Seismology Division,
Dominion Observatory,

D. H. WeICHERT

Ottawa, Canada.

Received February 7,1969

1 Lilwall, R. C., and Douglas, A., Nature,220, 362 (1968).

2 wero, Geophys, J. R. Astro. eichert, D. H., Manched
Soc., 13, 277 (1967).

${ }^{3}$ Blamey, C., and Gibbs, 1. G., Geophys. J. R. Astro. Soc., 16, 1 (1968). 